\title{
BRANDING FRESH FOOD: WHO IS WILLING TO PAY MORE FOR BEEF?
}

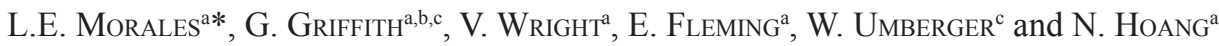 \\ aUNE Business School, W40 EBL Building, Armidale Campus, University of New England, Armidale, \\ New South Wales 2351. Australia \\ ${ }^{b}$ Faculty of Veterinary and Agricultural Sciences, 142 University of Melbourne, University Street, Parkville, \\ Victoria 3053. Australia \\ ${ }^{\mathrm{c}}$ Global Food Studies Program, Faculty of the Professions, 5.05 NEXUS 10 Tower, The University of Adelaide, \\ South Australia 5005. Australia
}

(Received: 17 October 2015; accepted 17 December 2015)

Retail beef markets not offering objective grade indicators in-store leave consumers uncertain about the quality of beef they purchase. The introduction of fresh brands can better meet consumers' expectations. Willingness-to-pay for 'ideal' quality cuts represents the maximum premiums brands could achieve. Using Australian consumers' survey data, the maximum premiums are modelled using zero-inflated negative binomial models. Results indicate that buyer's characteristics and perceptions about product attributes influence the premiums for high-quality beef that consumers are willing to pay for a cut considered 'ideal', indicating the maximum premium that brands could achieve in relevant segments. The premiums indicate there is substantial potential for beef cuts that reduce purchase risk by more assuredly meeting buyer's preferences in Australia.

Keywords: fresh beef, consistent high-quality, branded food, willingness-to-pay

Consumers can experience problems predicting the quality of fresh food products in-store, when their inferences are based only on the appearance of the product and other quality cues (ZeithamL, 1988). Misconceptions about quality cues can produce differences between expected and experienced quality, leading to high perceived risk among consumers when they make a purchase choice (HENCHION et al., 2014). Branding can be useful in such circumstances when the brand is considered to be a reliable predictor of valued product attributes (Zeithaml, 1988; Morrison \& EASTBurn, 2006).

Previous research identified correlations between willingness-to-pay for certified eatingquality beef, and customer age (LYFORD et al., 2010); location (central, southern and western regions in the United States), race (white), marital status, household size, gender (female), and age (MARTinez et al., 2007); and frequency of beef consumption and male gender (Froehlich et al., 2009). In contrast, income was not found to be a significant predictor of willingness to pay for branded beef by MARTINEZ and co-workers (2007) and by FroEHLICH and co-workers (2009), who also found gender and education non-significant variables. In addition, willingness-to-pay has been found for specific attributes of fresh beef, including animal feeding method (XUE et al., 2010), production process, taste and health information (UMBERGER et al., 2009; SEPÚLVEDA et al., 2013); naturalness, guaranteed tenderness and breed (Froehlich et al., 2009); country of origin (Loureiro \& UMBerger, 2003; SzakÁly et al., 2015); and brand, colour, and marbling (Morrison \& EASTBURn, 2006).

\footnotetext{
* To whom correspondence should be addressed.

Phone: +61 26773 2031; fax: +61 26773 3596; e-mail: emilio.morales@une.edu.au
} 
A meat quality evaluation system called Meat Standards Australia (MSA) has been implemented to offer guaranteed levels of eating-quality to beef consumers (PoLKINGHORNE et al., 2008). According to Griffith and ThOMPSON (2012), price premiums for labelled MSA cuts were $0.30 \mathrm{c} \mathrm{kg}^{-1}$ at the retail level between the period 2004/05 and 2010/11 on a whole carcass basis. Currently, the use of MSA grades by supermarkets has been to provide quality consistency, but it has not been used to offer a range of differentiated beef cuts. Despite the premiums paid for MSA cuts, most retailers have introduced quality differentiation mainly based on fat content, breed, animal feeding, and origin. There are some fresh beef brands offered on the basis of regional and breed attributes. They are poorly represented in the major supermarkets and constrained to deliver consistent quality.

This research studies the variables that affect the maximum premium consumers are willing to pay for fresh beef cuts that match their expectations, which represents the premium that differentiated branded products could achieve. The present study focuses on those consumers classified in the Potential Target Market (PTM), who are in medium-to-high income segments and, therefore, the most likely to be willing to pay higher premiums for an 'ideal' high-quality branded product.

\section{Materials and methods}

\subsection{Data}

An online survey interviewed residents across Australia who were over 18 years old and decided the beef purchased for their households during January 2010. The preferences of all beef consumers are expected to be represented by the beef buyers as they are presumed to shop for cuts that would be acceptable to household members. Respondents were randomly selected from members of a panel of approximately 300000 people, resulting in 1084 completed questionnaires. They were individually invited to participate in the survey and care was taken to keep a proportion of respondents close to the characteristics of the Australian population to avoid a potential source of bias. The sample size was estimated considering 95 percent confidence level and a 3 percent margin error. The main characteristics of the sample are shown in Table 1.

Beef buyers were grouped in six segments through agglomerative hierarchical clustering using the variables collected with the survey, including demographics, behaviours, preferences, interests, and psychographics. Based on the characteristics of the groups, those that have medium to high income and have an appreciation for high-quality beef were selected as the PTM for branded beef. The three groups selected include the segments: 'high involvement and high income', 'low knowledge, busiest, and highest income' and 'high quality concerned and medium income'.

\subsection{Willingness to pay model}

The utility function in attributes space models consumers with different characteristics and perceptions about attributes used to predict quality dimensions (Tonsor et al., 2005):

$$
U_{\mathrm{i}}(a)=\alpha+\beta A_{i}+\gamma C_{i}+\varepsilon_{i}
$$

where $U_{\mathrm{i}}(a)$ is the consumer's utility function in attributes space; $\alpha$ is a constant for beef products, associated with the consumer's intrinsic preferences; $A_{\mathrm{i}}$ is a vector of perceptions 
about attributes used as quality cues; $\beta$ is a vector that gives the weight of each product attribute; $C_{\mathrm{i}}$ is a vector of the characteristics and influences of the individual consumer $i$; and $\gamma$ is a vector that indicates the degree of influence of these characteristics on the utility perceived by consumer $i$ from beef products. Finally, $\varepsilon_{\mathrm{i}}$ is a stochastic error component.

Table 1. Demographic characteristics of the respondents

\begin{tabular}{|c|c|c|c|}
\hline Variable & Percentage & Variable & Percentage \\
\hline Gender & & Children & \\
\hline Male & 49 & No & 64 \\
\hline Female & 51 & Yes & 36 \\
\hline Age & & Occupation & \\
\hline 18 to 24 years old & 11 & Working full-time & 43 \\
\hline 25 to 34 years old & 21 & Working part-time & 20 \\
\hline 35 to 44 years old & 20 & Full-time student & 3 \\
\hline 45 to 49 years old & 11 & Part-time student & 1 \\
\hline 50 to 54 years old & 10 & Working and studying & 2 \\
\hline 55 to 64 years old & 14 & Retired & 18 \\
\hline Over 65 years old & 14 & Full-time home duties & 6 \\
\hline Living area & & Looking for a job & 3 \\
\hline Northern Territory & 1 & On a pension & 4 \\
\hline Australian Capital Territory & 3 & Education level & \\
\hline New South Wales & 31 & Primary school (or some) & 1 \\
\hline Victoria & 25 & Some secondary school & 17 \\
\hline Queensland & 20 & Finished year 12 & 20 \\
\hline South Australia & 8 & Diploma from CAE/TAFE & 26 \\
\hline Western Australia & 10 & Graduate degree UNI/TAFE & 26 \\
\hline Tasmania & 3 & Postgraduate degree & 10 \\
\hline Living with partner & & Income level (before taxes) & \\
\hline No & 36 & Below $\$ 20,000$ & 9 \\
\hline Yes & 64 & $\$ 20001$ to 40000 & 17 \\
\hline Household size & & $\$ 40001$ to 60000 & 18 \\
\hline 1 person & 11 & $\$ 60001$ to 80000 & 16 \\
\hline 2 people & 36 & $\$ 80001$ to 100000 & 14 \\
\hline 3 people & 20 & $\$ 100001$ to 120000 & 11 \\
\hline 4 people & 19 & $\$ 120001$ to 150000 & 7 \\
\hline 5 people & 9 & $\$ 150001$ to 200000 & 6 \\
\hline 6 people & 4 & Over $\$ 200000$ & 3 \\
\hline 7 people & 1 & & \\
\hline 8 people & 0 & & \\
\hline 9 people & 0 & & \\
\hline
\end{tabular}


The extra willingness-to-pay for a differentiated product will be a function of the differences in perceived attributes, income, time constraints, and preferences based on a consumer's characteristics and influences (FroenLich et al., 2009):

$$
W T P_{\mathrm{i}}(x)=f\left(A_{\mathrm{i}}, I_{\mathrm{i}}, t_{\mathrm{i}}, P R_{\mathrm{i}}\right)
$$

where $W T P_{\mathrm{i}}$ is the willingness of individual $i$ to pay extra for a beef product; $x$ represents a high-quality beef product; $A_{\mathrm{i}}$ is a vector of perceptions about beef attributes of consumer $i$; $I_{\mathrm{i}}$ is the income of individual $i ; t_{\mathrm{i}}$ represents the time constraints of consumer $i$ (related to shopping convenience); and $P R_{\mathrm{i}}$ is a vector of preferences of individual $i$ (influenced by preference shifters).

Given the nonnegative characteristics of the maximum extra willingness-to-pay, truncated below zero dollars, over-dispersed and with excess of zeros, due to consumers that are not willing-to-pay premiums, zero-inflated negative binomial models were applied. The nonnegative condition makes estimation by conventional ordinary least squares inappropriate, while the over-dispersion of the dependent variable can overstate the significance of coefficients obtained through Poisson regression. If a buyer is willing to pay extra for a high quality branded beef cut (and hence is convinced about the quality of the product), the probability that the buyer would pay a premium $y_{\mathrm{i}}$ is given by:

$$
\operatorname{Pr}\left(y_{i} \mid z_{i}\right)=\frac{\Gamma\left(y_{i}+\alpha^{-1}\right)}{y_{i} ! \Gamma\left(\alpha^{-1}\right)}\left(\frac{\alpha^{-1}}{\alpha^{-1}+\mu_{i}}\right)^{\alpha^{-1}}\left(\frac{\mu_{i}}{\alpha^{-1}+\mu_{i}}\right)^{y_{i}}
$$

where $y_{\mathrm{i}}$ is the extra willingness-to-pay for an 'ideal' branded beef cut of individual $i, z_{\mathrm{i}}$ represents a specific explanatory variable for individual $i, \Gamma$ is the gamma function, $\alpha$ is the over-dispersion coefficient, and $\mu_{\mathrm{i}}$ is equal to $\exp \left(z_{\mathrm{i}} \beta\right)$ (LONG \& FreEse, 2014).

There are two latent or unobserved groups considered in the zero-inflated negative binomial model that are identified through the logistic component. The first group is composed of individuals that are never willing to pay premiums for branded beef, while the second group is composed of buyers whose willingness-to-pay extra might be zero, but there is a probability that they could be willing to pay premiums for branded beef. The logistic component of the zero-inflated negative binomial models predicts the probability of a respondent being in the group that is never willing to pay a premium for an 'ideal' cut compared with those that would be willing to pay extra. The negative binomial component presents coefficients for the change in the expected premium, using only information of those beef buyers that would be willing to pay extra. This model requires to be applied using a dataset that contains latent groups, in the case under study those respondents that are always willing to pay zero for branded beef; otherwise we could get biased estimations. Given the characteristics of the dataset used in this study, we observe the presence of two latent groups, which supports the use of this model (LONG \& FrEESE, 2014). 


\section{Results and discussion}

Multiple regressions using zero-inflated negative binomial models were estimated for: (1) all respondents included in the PTM excluding those who would not buy branded beef (all target beef buyers (TBB-All)); and (2), only those who would buy branded beef (TBB-Yes). The average extra willingness-to-pay per kilogram is $\$ 5.00$ for all target beef buyers (TBB-All) and $\$ 5.37$ for those willing to buy branded beef (TBB-Yes). This variable is over-dispersed as its variance is $\$ 29.70$ for all target beef buyers and $\$ 31.02$ for those who would buy branded beef. In addition, excesses of zeros were found in both estimations on the basis of the VuOng (1989) test, with P-values lower than 0.01. The Hausman (1978) endogeneity test determined that all variables included in the model can be considered as exogenous, so there were no problems of endogeneity. Also, collinearity diagnostic tests developed by BELSLEY (1982) rejected the presence of degrading collinearity.

Estimates of the significant variables included in the willingness-to-pay models for TBB-All and TBB-Yes are presented in Table 2. Estimated incidence rate ratios (IRRs) are included in the negative binomial components (willingness-to-pay premiums) to demonstrate the sensitivity of extra willingness-to-pay to each factor. An IRR greater than unity indicates a positive impact of a factor on extra willingness-to-pay, while a ratio below one indicates a negative effect (LONG \& FREESE, 2014).

The three variables included in the logistic component (which analyses the variables affecting the propensity to do not pay premiums) of the model for TBB-All have significant estimated coefficients, while in the TBB-Yes model none of the estimated coefficients is significant. This is explained by the proportion of respondents not willing to pay extra. In the case of TBB-All, the positive effect of working full-time on the probability of being included in the group unwilling to pay a premium can be explained by time constraints to find a highquality product that justifies paying extra, while the negative effect of previous experience with branded beef demonstrates that most respondents who have bought branded beef are prepared to pay premiums for high-quality beef. The positive effect of the psychographic factor 'Quality consistency' on the probability of being unwilling to pay a premium indicates that those target beef buyers concerned about quality consistency are looking for a product with a guaranteed consistent quality at an acceptable level instead of a high-quality level.

The variables that affect positively the premiums for an 'ideal' branded beef cut in both models by impact order indicated by the IRRs, are: i) membership of cluster 'High qualityconcerned and medium income'; ii) 'Involvement, beef quality, and price as indicator'; iii) 'Quality consistency'; iv) 'Credence attributes'; v) level of agreement that branded beef is worth more than unbranded beef; and vi) household income. The psychographic factor 'Instore information and assistance' reduced the willingness-to-pay premiums for 'ideal' branded beef products. In the case of 'Age' and 'Never shopping at supermarkets', they are significant variables only in the TBB-All model. In both models, cluster membership of 'High quality-concerned and medium income' has one of the highest positive effects on willingness-to-pay (IRR above 1.6) compared to the control cluster 'Low beef knowledge, busiest, and highest income', while household income has the lowest positive effect (IRR just above unity) among the significant variables. This result demonstrates a higher relevance of concern for quality than income for the potential premium of beef cuts, which confirms the results reported by MARTINEZ and co-workers (2007) and by FroEHLICH and co-workers (2009). 
Analysing the results of the negative binomial component of the model for TBB-All, the binary variable 'Never shop for beef at supermarkets' has the strongest positive effect on the willingness-to-pay, closely followed by membership of the cluster 'High quality-concerned and medium income'. These results suggest that those beef buyers who are concerned about high quality, and those who buy beef somewhere other than in supermarkets, are looking for high-quality cuts and are prepared to pay higher premiums for branded cuts. Thus, a beef buyer who considers price to be a good indicator of quality, has a high knowledge about beef and appreciates 'credence attributes' of the product (which include certifications and branding), is willing to pay a higher premium. These results demonstrate that knowledge and appreciation of high-quality branded cuts increase the premiums to be paid.

Appreciation of branded beef and quality consistency also increases the premiums for branded cuts. The significance of quality consistency in the premium for branded beef demonstrates that those target beef buyers willing to pay extra appreciate consistent high quality, in contrast to those unwilling to pay premiums as they require beef quality of only an acceptable level. This result confirms the findings reported by FroeHLICH and co-workers (2009), Umberger and co-workers (2009), Xue and co-workers (2010) and Sepúlveda and co-workers (2013). Finally, household income also increases the premiums for branded beef, which confirms the findings reported by FroEHLICH and co-workers (2009). The negative effect of age on willingness-to-pay for branded beef products also confirmed the results reported by Froenlich and co-workers (2009), and LyFord and co-workers (2010), who identified negative or uncertain effects on willingness-to-pay.

\section{Conclusions}

Quality appreciation has a higher impact than income on the potential premium for consistent high-quality beef cuts among medium-to-high income beef consumers. These results confirm those reported by FroenLich and co-workers (2009) and LYFORD and co-workers (2010), where income was found to be non-significant or its effects uncertain. The factors increasing the willingness-to-pay extra for branded beef products include: i) appreciation of branded beef; ii) concern about high quality, quality consistency, and credence attributes; iii) knowledge about beef; iv) the perception that price is a good indicator of quality; and v) lack of requested information and assistance at the purchase point.

Beef buyers included in the PTM are willing to pay $\$ 5.00 / \mathrm{kg}$ extra on average for an 'ideal' cut of beef, which is higher than the average retail premium for specific cuts with generic labels of MSA grades reported by GRIFFITH and THOMPSON (2012). Given the data was collected during the global financial crisis, the willingness-to-pay premiums for high-quality beef might be lower than the one that could prevail nowadays. Therefore, there is potential for the development of differentiated consistent high-quality guaranteed beef cuts according to the preferences of different consumer segments in Australia, which confirms the potential to further differentiate beef products presented by GRUNERT and co-workers (2004) and Polkinghorne and co-workers (2008). Hence, further studies about potential branded products aiming to different consumer niches are required to identify those that better meet consumers' expectations, and consequently, the beef industry would be able to capture the maximum consumers are willing-to-pay for 'ideal' beef cuts. In addition, retailers should study the effects on willingness-to-pay premiums when they provide more explicit and 
objective quality information derived from MSA grading to customers, as both producer and consumer welfare are expected to be enhanced.

The authors acknowledge the contribution of the Australian Cooperative Research Centre for Beef Genetic Technologies (Beef CRC) for providing data and valuable comments. The authors also thank to the participants of the Annual Conference of the Australian Agricultural and Research Economics Society (AARES) for their valuable comments.

\section{References}

BeLSLEY, D. (1982): Assessing the presence of harmful collinearity and other forms of weak data through a test for signal-to-noise. J. Econometrics, 20, 211-253.

Froehlich, E., Carlberg, J. \& Ward, C. (2009): Willingness-to-pay for fresh brand name beef. Can. J. Agr. Econ. $57,119-137$.

Griffith, G.R. \& Thompson, J.M. (2012): The aggregate economic benefits to the Australian beef industry from the adoption of meat standards Australia: updated to 2010/11. Australas. Agribusiness Rev., 20, 11-38.

Grunert, K.G., Bredahl, L. \& Brunso, K. (2004): Consumer perception of meat quality and implications for product development in the meat sector - A review. Meat Sci., 66(2), 259-272.

Hausman, J.A. (1978): Specification tests in econometrics. Econometrica, 46, 1251-1271.

Henchion, M., McCarthy, M., Resconi, V. \& Troy, D. (2014): Meat consumption: trends and quality matters. Meat Sci., 98(3), 561-568.

LonG, J. \& Freese, J. (2014): Regression models for categorical dependent variables using Stata. Texas: Stata Press, College Station, $3^{\text {rd }}$ ed., p. 589.

Loureiro, M. \& UMBERGER, W. (2003): Estimating consumer willingness-to-pay for country-of-origin labelling. J. Agr. Resour. Econ., 28(2), 287-301.

Lyford, C., Thompson, J., Polkinghorne, R., Miller, M., Nishimura, T., Neath, K., Allen, P. \& Belasco, E. (2010): Is willingness to pay (WTP) for beef quality grades affected by consumer demographics and meat consumption preferences? Australas. Agribusiness Rev., 18, 1-17.

Martinez, S., Hanagriff, R., Lau, M.. \& Harris, M. (2007): Factors affecting demand for branded beef. Southern Agr. Econ. Assoc., Annual Meeting. Available at the website: ageconsearch.umn.edu/bitstream/34885/1/ sp07ma01.pdf. (last accessed: 17 December 2015)

Morrison, M. \& EASTBURn, M. (2006): A study of brand equity in a commodity market. Australas. Marketing $J$. (AMJ), 14(1), 62-78.

Polkinghorne, R., Thompson J.M., Watson, R., Gee, A. \& Porter, M. (2008): Evolution of the Meat Standards Australia (MSA) beef grading system. Aust. J. Exp. Agr., 48, 1351-1359.

Sepúlveda, W.S., Maza, M.T. \& Mantecón, A.R. (2013): Information demanded by consumers: New issues in voluntary beef labelling. Acta Alimentaria, 42, 135-142.

SzakÁly, Z., Soós, M., Szabo, S. \& Szente, V. (2015): Role of labels referring to quality and country of origin in food consumers' decisions. Acta Alimentaria, doi: 10.1556/AAlim.2015.0012.

Tonsor, G.T., Schroeder, T.C., Fox, J.A. \& Biere, A. (2005): European preferences for beef steak attributes. J. Agr. Resour. Econ., 30, 367-380.

UMBERGER, W., BOXALL, P. \& LACY, R. (2009): Role of credence and health information in determining US consumers' willingness-to-pay for grass-finished beef. Aust. J. Agr. Resour. Ec., 53, 603-623.

Vuong, Q.H. (1989): Likelihood ratio test for model selection and non-nested hypotheses. Econometrica, 57, $307-$ 333.

XuE, H., Mainville, D., You, W. \& NAYga, R.M. JR. (2010): Consumer preferences and willingness to pay for grassfed beef: Empirical evidence from in-store experiments. Food Qual. Prefer., 21, 857-866.

Zeithaml, V.A. (1988): Perceptions of price, quality, and value: A means-end model and synthesis of evidence. J. Marketing, 52(3), 2-22. 